\title{
A plasmaspheric mass density model and constraints on its heavy ion concentration
}

\author{
D. Berube ${ }^{1}$, M.B. Moldwin ${ }^{1,2}$, S.F. Fung ${ }^{3}$, J.L. Green ${ }^{3}$
}

1. UCLA Department of Earth and Space Sciences, Los Angeles, CA

2. UCLA Institute of Geophysics and Planetary Physics, Los Angeles, CA

3. NASA Goddard Space Flight Center, Greenbelt, MD

Submitted to Journal of Geophysical Research Letters

July 2,2004

\begin{abstract}
The first empirical model of the equatorial mass density of the plasmasphere is constructed using ground-based ULF wave diagnostics. Plasmaspheric mass density between $L=1.7$ and $L=3.2$ has been determined using over 5200 hours of data from pairs of stations in the MEASURE array of ground magnetometers. The least-squares fit to the data as a function of $L$ shows that mass density falls logarithmically with $L$. Average ion mass as a function of $L$ is also estimated by combining the mass density model with plasmaspheric electron density profiles determined from the IMAGE Radio Plasma Imager (RPI). Additionally, we use the RPI electron density database to examine how the average ion mass changes under different levels of geomagnetic activity. We find that average ion mass is greatest under the most disturbed conditions. This result indicates that heavy ion concentrations are enhanced during large geomagnetic
\end{abstract}


disturbances, and therefore play an important role in storm-time plasmaspheric dynamics. The average ion mass is also used to constrain the concentrations of $\mathrm{He}^{+}$and $\mathrm{O}^{+}$. Estimates of the $\mathrm{He}^{+}$concentration determined this way can be useful for interpreting IMAGE Extreme Ultraviolet Imager (EUV) images.

\section{Introduction}

Many studies have been performed using spacecraft data and VLF whistlers to determine the equatorial electron density profile of the inner magnetosphere [Chappell, 1972, Farrugia, 1989, Carpenter and Anderson, 1992, Fung et al., 2001, Sheeley et al., 2001]. The usual result is that plasmaspheric equatorial electron density $\left(n_{e}\right)_{e q}$ ranges from hundreds to many thousands per cubic centimeter and decreases as a power law with radial distance from the Earth. Diurnal [Park, 1978], annual [Clilverd et al, 1991], and storm-time [Park, 1973; Park, 1974] variations of the total electron plasma density have all been observed.

The mass density and composition of the plasmasphere are not as well known, particularly the inner plasmasphere $(\mathrm{L}<3)$. This is because satellites pass through this region quickly and spacecraft charging effects make direct measurements of the dense, low-energy plasma difficult [Moldwin, 1997]. Most of the mass density and composition observations of the plasmasphere have come from the DE 1, GEOS 1 and 2, and ISEE 1 missions [Horwitz et al., 1984, Farrugia et al., 1989]. These studies have shown that $\mathrm{He}^{+}$and $\mathrm{O}^{+}$are the major heavy ion species in the plasmasphere and usually have concentrations of about $2-6 \%$ for $\mathrm{He}^{+}$and $1 \%$ for $\mathrm{O}^{+}$. Craven et al. [1997] reported typical He+ to $\mathrm{H}+$ ratios of $\sim 0.03$ to 0.3 . Horwitz et al. [1984] found that in the aftermath of a storm $\mathrm{O}+$ density could become comparable to $\mathrm{H}+$ density in the plasmasphere. 
Techniques for remotely sensing the mass density along closed magnetic field lines involve using pairs of ground-based magnetometers to measure field line resonance frequencies. These frequencies can then be used to infer the mass density along the magnetic field, much like the mass density along a string fixed at both ends can be determined from its harmonic frequencies [Schultz, 1996; Denton and Gallagher, 2000]. Two of these techniques are the power ratio technique [Baransky et al., 1985, 1989] and the cross-phase technique [Waters et al., 1991, 1994]. Berube et al. [2003] have developed an automated method for determining field line eigenfrequencies using pairs of ground magnetometers. This new routine allows the analysis of large amounts of data, and hence the development of a mass density database for the plasmasphere.

In this study, a model of the equatorial mass density of the plasmasphere using ULF field line eigenfrequencies is constructed using data from pairs of ground-based magnetometers. The mass density model is then combined with the IMAGE RPI electron density model to determine the average ion mass and estimate the maximum concentration of heavy ions in the plasmasphere.

\section{Equatorial Mass Density Model}

The MEASURE array of ground magnetometers [Moldwin et al., 2004, http://measure.igpp.ucla.edu] is located along the east coast of the United States and consists of ten stations covering $L=1.7$ to $L=3.2$. Data from seven of the stations were used in this study. Field line eigenfrequencies have been determined using the method of Berube et al. [2003] for approximately 5200 hours of data from six pairs of stations in the array from the years 1999-2001 (The station pairs straddle $L=1.74,1.99,2.30,2.57,2.89$ and 3.12). The uncertainty associated with the selected frequencies is typically $1-2 \mathrm{mHz}$, 
and is discussed in detail by Berube et al. [2003]. Hourly averaged mass density is computed from the eigenfrequencies by assuming $\mathrm{ar}^{-3}$ dependence of density along the field line and using the results of Schulz [1996], who provides a relationship between field line resonant frequencies and mass density. Field line resonances are primarily present only during the daytime due to the reduced conductivity of the nightside ionosphere. Therefore, this model is only valid on the dayside.

Figure 1 shows the hourly mass density estimates obtained for each of the six pairs of magnetometers, plotted as a function of $L$. The line is the least squares linear fit to the $\log$ average density at each $L$. It is given by the expression

$$
\_ \text {eq }(L)=10^{-0.67 \mathrm{~L}+5.1} \quad 1.7<=L<=3.2
$$

The equation represents the average mass density of the dayside plasmasphere since it includes all levels of activity. For quiet times,

$$
\begin{array}{r}
{ }_{\text {eq }}(L)=10^{-0.65 \mathrm{~L}+5.1} \quad 1.7<=L<=3.2 \quad \\
-9<D s t<-3 \mathrm{nT}
\end{array}
$$

For the most disturbed times $(D s t<-100 \mathrm{nT})$

$$
\begin{aligned}
& \text { _eq }=10^{-0.74 \mathrm{~L}+5.5} \quad 1.7<=L<=3.2 \\
& \text { Dst }<-100 \mathrm{nT}
\end{aligned}
$$

\section{Radio Plasma Imager Electron Density}


The Radio Plasma Imager (RPI) [Reinisch et al., 2001] on board the IMAGE satellite makes in situ measurements of magnetospheric electron density. Using all RPI equatorial electron density measurements from May, 2000 through May, 2001, Fung et al. [2001] have constructed electron density profiles for various conditions and local times. The expressions for electron density were determined by first binning the data by $L$, and then performing a least squares fit to the binned data. For all the data,

$$
\mathrm{n}_{\mathrm{eq}}=10^{-0.66 \mathrm{~L}+4.89} \quad 2<=L<=3.5
$$

For quiet times,

$$
\begin{array}{ll}
\mathrm{n}_{\mathrm{eq}}=10^{-0.51 \mathrm{~L}+4.56} & 1.7<=L<=3.2 \\
& -9<\text { Dst }<-3 \mathrm{nT}
\end{array}
$$

and for disturbed times,

$$
\begin{array}{ll}
\mathrm{n}_{\mathrm{eq}}=10^{-1.04 L+5.41} & 2<=L<=3.5 \\
& \text { Dst }<-100 \mathrm{nT}
\end{array}
$$

\section{Average Ion Mass and Composition of the Plasmasphere}

The average ion mass can be determined from the mass and electron density profiles since $M_{\text {avg }}=\_$eq $/ n_{\text {eq }}$. Figure 2 is a plot of the average ion mass determined from the mass density and electron density models described above. The black line is the average ion mass found using all the data. The red and blue lines are the quiet and disturbed time profiles, respectively. The lighter weighted lines represent the uncertainty associated with each quantity, and are based on the uncertainties in the parameters of the 
least-squares fit to the data. The overall and quiet time profiles are close to unity for $2<$ $L<3$. On average, the plasmasphere is made up of mostly $\mathrm{H}^{+}$. For the most disturbed times $(D s t<-100 \mathrm{nT})$ the average ion mass is significantly larger, and increasing with increasing $L$. During storms, a considerable number of heavy ions are present in the plasmasphere, with more heavy ions at larger $L$, indicating the presence of a heavy ion torus in the plasmasphere. Since the major constituents of the plasmasphere are $\mathrm{H}^{+}, \mathrm{He}^{+}$, and $\mathrm{O}^{+}$, their concentrations can be estimated with the following relationship:

$$
M_{\text {avg }} \cong m_{H^{+}}\left[\% H^{+}\right]+m_{\mathrm{He}^{+}}\left[\% \mathrm{He}^{+}\right]+m_{\mathrm{O}^{+}}\left[\% \mathrm{O}^{+}\right]
$$

If only one heavy ion species is assumed to be present, we can determine the maximum concentration of that species. For example, the upper limit on the average ion mass at $L=2$ overall is approximately $2.2 \mathrm{amu}$, which implies a maximum $\mathrm{He}^{+}$concentration of $40 \%$ by number and a maximum of $8 \%$ for $\mathrm{O}^{+}$. Table 1 shows maximum heavy ion concentrations determined this way for the entire data set, quiet times, and disturbed times. The maximum percentage is determined from the upper limit on average ion mass in each case. It should be stressed that this maximum determination only assumes one heavy ion species. For example, $>100 \% \mathrm{He}^{+}$concentration infers that there must be heavier ion species present in order to explain the observed average ion mass.

\section{Discussion}

The $\mathrm{He}^{+}$and $\mathrm{O}^{+}$concentrations in Table 1 are estimates based on the average mass and electron densities of the plasmasphere. The findings presented here agree well with the results of previous studies. For example, Craven et al. [1997], using DE1/RIMS data, found $\mathrm{He}^{+} / \mathrm{H}^{+}$ratios of $\sim 0.03-0.3$ (roughly 3-25 percent if we assume no other heavy ions are present). They also found that the relative abundance of $\mathrm{He}^{+} / \mathrm{H}^{+}$decreases with 
distance from the Earth. Our findings are fairly consistent with both these results, particularly for the quiet-time plasmasphere. Recently, Dent et al. [2003], using the same ground techniques as this study combined with simultaneous electron density measurements, for a single case study, found quiet-time $\mathrm{He}^{+}$concentrations of 35-64 percent and $\mathrm{O}^{+}$concentrations of $7-13$ percent for $\mathrm{L}<3.45$, and almost no heavy ions for $\mathrm{L}>3.45$. Our average results are slightly lower than their single event estimates, but the trend of less heavy ions at higher $L$ for quiet times is the same.

The increase in heavy ion concentrations during disturbed times has interesting implications for ring current and radiation belt dynamics. In particular, the results show that during severe storms $(D s t<-100 \mathrm{nT})$, the plasmasphere is dominated by heavy ions, rather than $\mathrm{H}^{+}$.

Finally, independent estimates of the abundance of $\mathrm{He}^{+}$in the magnetosphere are a necessary aid to the inversion of IMAGE Extreme Ultraviolet (EUV) images. Information about the mass density along the line-of-sight is necessary to accurately determine the $\mathrm{He}^{+}$density distribution in the plasmasphere. Clilverd et al. [2003] found for two events that changes in the $\mathrm{He}^{+}$column abundance appear to correlate with changes in $\mathrm{He}^{+}$determined from ground-based VLF and ULF observations. Future studies will combine ground-based ULF wave computations of mass density with in situ and VLF-derived electron densities and IMAGE EUV $\mathrm{He}^{+}$observations to create a more complete picture of the plasmasphere.

\section{Acknowledgments}


The MEASURE array is supported by NSF grant ATM-01-9223 and data are available at http://measure.igpp.ucla.edu. David Berube is supported by NASA Graduate Student Research Fellowship NASA NGT5-117. The research at GSFC was support by the IMAGE project. 


\section{References}

Baransky, L.N., J.E. Borovkov, M.B. Gokhberg, S.M. Krylov, and V.A. Troitskaya, High resolution method of direct measurement of the magnetic field lines' eigenfrequencies, Planetary Space Science, 33 (12), 1369, 1985.

Baransky, L.N., S.P. Belokris, Y.E. Borovkov, M.B. Gokhberg, N. Federov, and C.A. Green, Restoration of the meridional structure of geomagnetic pulsation fields from gradient measurements, Planetary Space Science, 37 (7), 859, 1989.

Berube, D., M.B. Moldwin, and J.M. Weygand, An automated method for the detection of field line resonance frequencies using ground magnetometer techniques, $J$. Geophys. Res., 108(A9), 1348, doi:10.1029/2002JA009737, 2003.

Carpenter, D.L., and R.R. Anderson, An ISEE/whistler model of equatorial electron density in the magnetosphere, J. Geophys. Res., 97 (A2), 1097, 1992.

Clilverd, M.A., A.J. Smith, and N.R. Thomson, The annual variation in quiet time plasmaspheric electron density, determined from whistler mode group delays, Planetary Space Science, 39 (7), 1059, 1991.

Clilverd, M.A., F.W. Menk, G. Milinevski, B.R. Sandel, J. Goldstein, B.W. Reinisch, C.R. Wilford, M.C. Rose, N.R. Thompson, K.H. Yearby, G.J. Bailey, I.R. Mann, and D.L. Carpenter, In-situ and ground-based intercalibration measurements of plasma density at L=2.5, J. Geophys, Res., 108(A10), 1365, doi: 10.1029/2003JA009866, 2003."

Craven, P.D., D.L. Gallagher, and R.H. Comfort, Relative concentration of $\mathrm{He}+$ in the inner magnetosphere as observed by the $\mathrm{DE} 1$ retarding ion mass spectrometer, $J$. Geophys Res., 102 (A2), 2279, 1997.

Dent, Z.C., I.R. Mann, F.W. Menk, J. Goldstein, C.R. Wilford, M.A. Clilverd, and L.G. Ozeke, A coordinated ground-based and IMAGE satellite study of quiet-time plasmasphere density profiles, Geophys. Res. Lett.,30, 12, doi: 10.1029/2003GL016946, 2003.

Denton, R.E., and D.L. Gallagher, Determining the mass density along magnetic field lines from toroidal eigenfrequencies, J. Geophys. Res., 105 (A12), 27717, 2000.

Farrugia, C.J., D.T. Young, J. Geiss, and H. Balsiger, The composition, temperature, and density structure of cold ions in the quiet terrestrial plasmasphere: GEOS 1 results, $J$. Geophys Res., 94 (A9), 11865, 1989.

Fung, S.F., L.N. Garcia, J.L. Green, D.L. Gallagher, D.L. Carpenter, B.W. Reinisch, I.A. Galkin, G. Khmyrov, and B.R. Sandel, Plasmaspheric electron density distributions 
sampled by radio plasma imager on the IMAGE satellite, Eos Trans, AGU, volume, page, 2001.

Horwitz, J.L., R.H. Comfort, and C.R. Chappell, Thermal ion composition measurements of the formation of the new outer plasmasphere and double plasmapause during storm recovery phase, Geophys. Res. Lett., 11 (8), 701, 1984.

Moldwin, M.B., Outer Plasmaspheric Properties: What we Know From Satellite Data, Space Science Reviews, 80, 181, 1997.

Park, C.G., Whistler Observations of the Depletion of the Plasmasphere during a Geomagnetic Substorm, Journal of Geophysical Research, 78 (4), 672, 1973.

Park, C.G., Some Features of Plasma Distribution in the Plasmasphere Deduced From Antarctic Whistlers, Journal of Geophysical Research, 79 (1), 169, 1974.

Park, C.G., D.L. Carpenter, and D.B. Wiggin, Electron Density in the Plasmasphere: Whistler Data on Solar Cycle, Annual, and Diurnal Variations, Journal of Geophysical Research, 83 (A7), 3137, 1978.

Reinisch, B.W., X. Huang, D.M. Haines, I.A. Galkin, J.L. Green, R.F. Benson, S.F. Fung, W.W.L. Taylor, P.H. Reiff, D.L. Gallagher, J.-L. Bougeret, R. Manning, D.L. Carpenter, and S.A. Boardsen, First results from the Radio Plasma Imager on IMAGE, Geophys. Res. Lett., 28 (6), 1167, 2001.

Schulz, M., Eigenfrequencies of geomagnetic field lines and implications for plasmadensity modeling, J. Geophys. Res., 101 (A8), 17385, 1996.

Sheeley, B.W., M.B. Moldwin, and H.K. Rassoul, An empirical plasmasphere and trough density model: CRRES observations, J. Geophys Res., 106 (A11), 25631, 2001.

Waters, C.L., F.W. Menk, and B.J. Fraser, Low latitude geomagnetic field line resonance: experiment and modeling, J. Geophys. Res., 99 (A9), 17547, 1994.

Waters, C.L., F.W. Menk, and B.J. Fraser, The resonance structure of low latitude pc3 geomagnetic pulsations, Geophys. Res. Lett., 18 (12), 2293, 1991. 


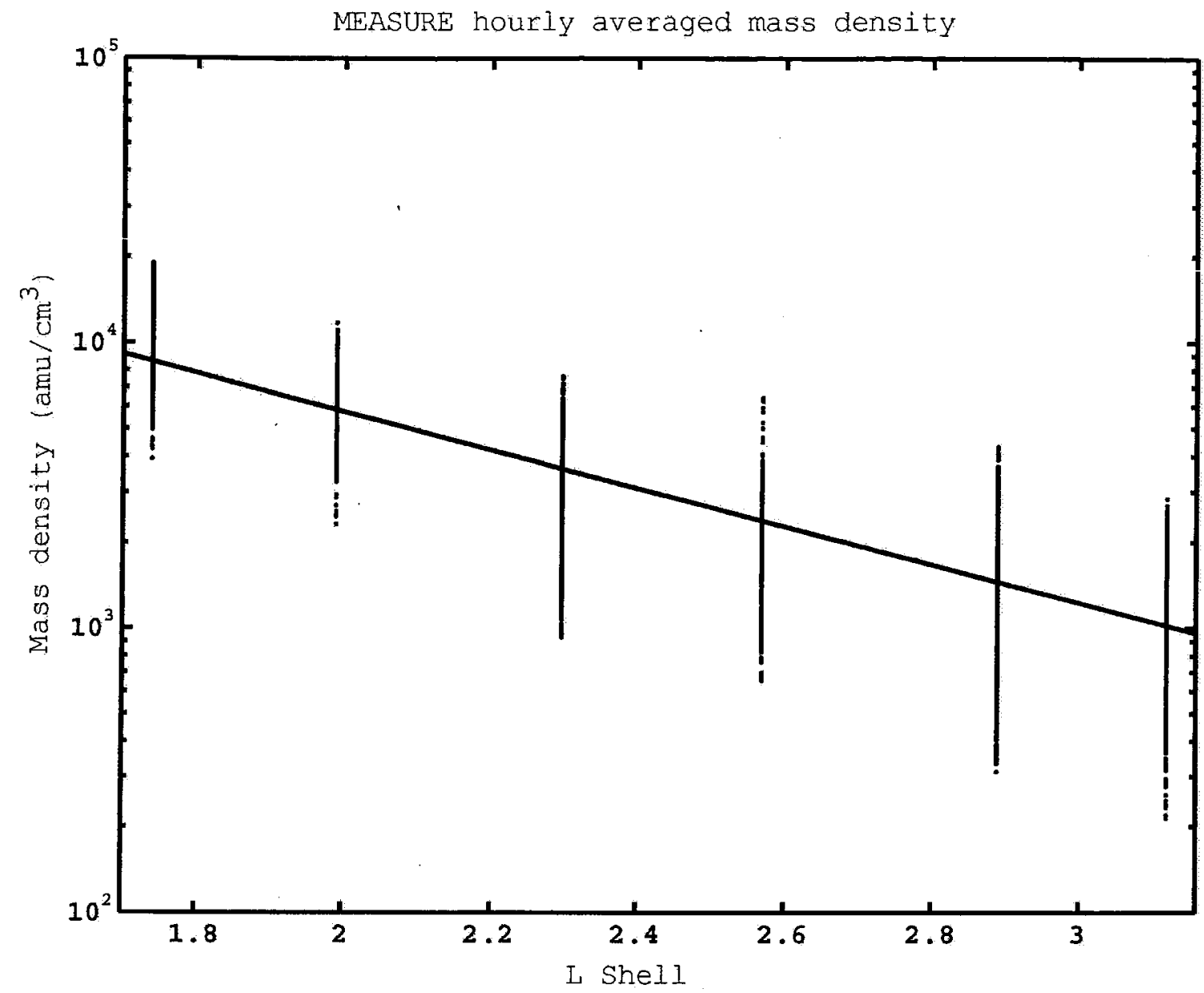

Figure 1. Average plasmaspheric mass density from 6 pairs of stations in the MEASURE array. The line represents the least squares linear fit to the mean density at each $L$ and is given by equation 1 in the text. 


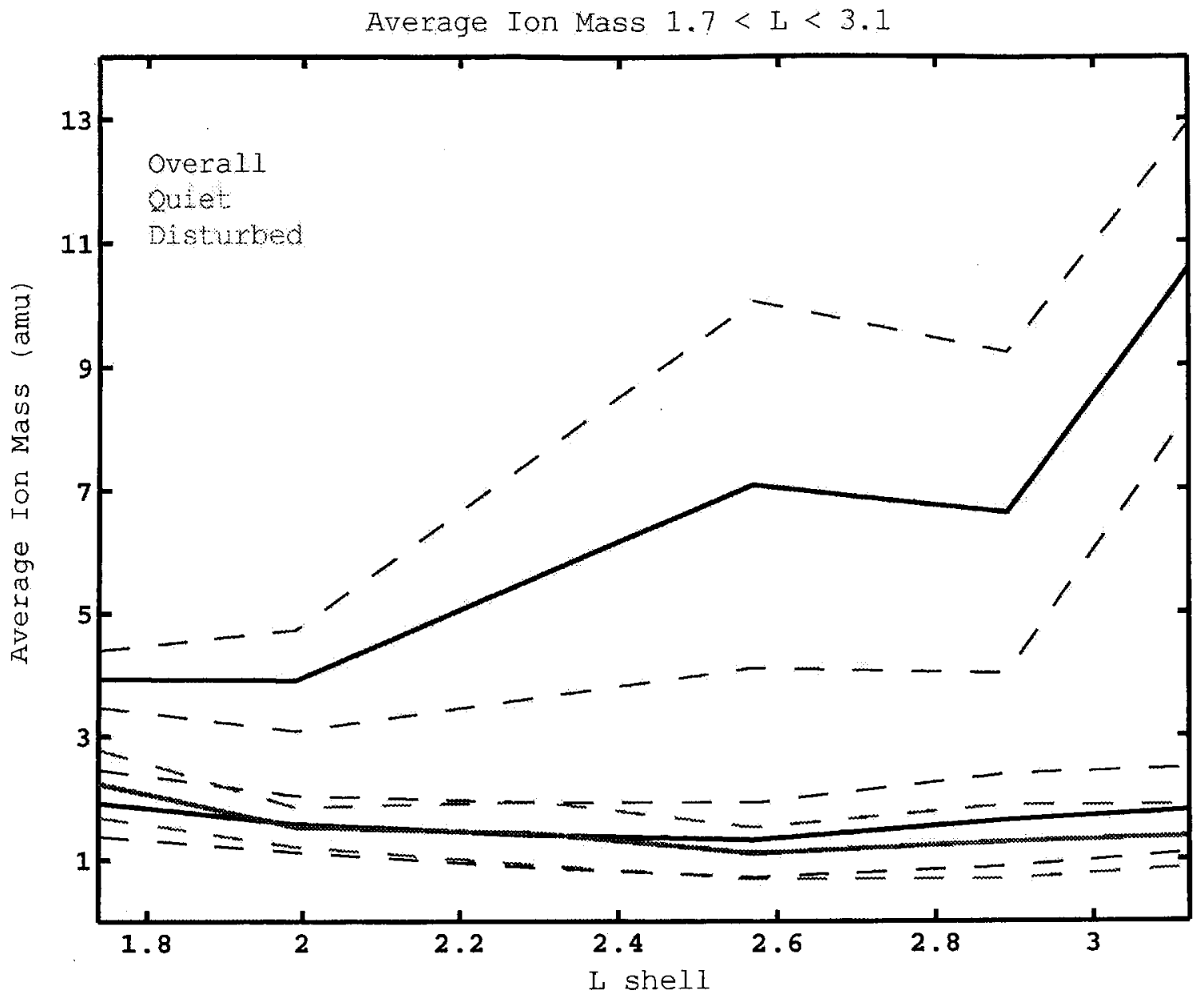

Figure 2. Average ion mass derived from equatorial mass and electron density models. The three lines represent the ion mass overall, low activity $(-9 \mathrm{nT}<D s t<-3 \mathrm{nT})$, and disturbed activity $\left(D s t_{3}<-100 \mathrm{nT}\right)$. The lighter dashed lines represent the uncertainty in each quantity, based on the uncertainties in the parameters of the least squares fit to the data. 


\begin{tabular}{|l|c|c|c|c|}
\hline & \multicolumn{2}{|c|}{$\mathrm{He}^{+}$} & \multicolumn{2}{c|}{$\mathrm{O}^{+}$} \\
\hline & $\mathrm{L}=2$ & $\mathrm{~L}=3$ & $\mathrm{~L}=2$ & $\mathrm{~L}=3$ \\
\hline All Data & $40 \%$ & $45 \%$ & $8.0 \%$ & $8.9 \%$ \\
\hline Quiet Times & $43 \%$ & $29 \%$ & $8.6 \%$ & $5.7 \%$ \\
\hline $\begin{array}{l}\text { Disturbed } \\
\text { Times }\end{array}$ & $100 \%$ & $100 \%$ & $25 \%$ & $62 \%$ \\
\hline
\end{tabular}

Table 1. Maximum heavy ion concentrations determined from ground-data-derived mass density model and RPI in situ electron density profiles, using equation (7) from the text, and assuming only one heavy ion species is present for each case. 